\title{
Part 7: Education, Learning and Intellectual Disability
}

\author{
Recommendations for Using Assistive Technologies \\ for Inclusive Media Education in Kindergartens \\ Saša Stepanovića ${ }^{\mathrm{a}, *}$, Veselin Medenica ${ }^{\mathrm{a}}$, Ivana Ristića \\ and Lidija Ivanovića \\ ${ }^{a}$ Deparment of Occupational Therapy, The College of \\ Social Work, Terazije 34, Belgrade, Serbia
}

Background: The role of assistive technologies (AT) in inclusive media education is a rarely discussed topic because it is considered that the use of ATs in kindergarten is premature. The potential of digital media for the participation of children with disabilities is big, but there is no data on how to use ATs and the media in such a way as to increase accessibility for children with disabilities in kindergarten. Therefore, we aim to identify issues of accessibility of content according to the 'Doing Disability Concept', to identify ways in which identified problems can be solved using ATs and ultimately to give recommendation for use of ATs in kindergartens to solve identified problems.

Method: A team of 3 experts, working with children in a kindergarten has been formed. It was composed by the teacher, special educator, occupational therapist in order to create recommendations for the creation and use of ATs in the kindergarten. In Phase 1, team had to define problems of accessibility to the content/ activities. Seventy-two papers were reviewed, using the Doing Disability Checklist, for recording identified problems. In Phase 2, it was necessary to review papers which explains the ways in which the ATs solve the problems identified in Phase 1. Of the 92 papers reviewed, 7 were identified that meet the above criterion. Based on the review, the experts completed a list of recommendations for use of AT in inclusive media education in kindergartens.

Key results: In Phase 1 problems of accessibility of content are identified as: non-use or lack of adequate technologies to ensure accessibility; inadequate design of existing technologies used and digital divide; lack of IT education for teachers; lack of practical ways for inclusion; lack of motivation for children with disabilities to get involved; rejection by the peers. The results of Phase 2. indicated the positive role of ATs in addressing the identified problems: 1. Impact on the motivation of the child for participation; 2. Compensation of developmental difficulties facilitates the inclusion and prevention of digital divide; 3 . Training is usually included when ATs are provided, which makes the problem of lack of teachers education solved.

Conclusion: Recommendations have been made for the use of ATs in kindergartens: Pedagogical staff should be technologically literate; When selecting ATs, make sure that all children can be involved in interaction through technology not only those with disabilities; Choose ATs which stimulate attention and child's play; AT should play a role in stimulating the development of skills, not only compensation; If technologies and media are not adapted for all children, make sure to use ATs or do not use technology at all. Include children in the design process of new ATs.

Keywords: Assistive Technology, Kindergarten, Inclusive Education.

${ }^{*}$ Corresponding author. E-mail: salenono86@gmail. com

Creating Appropriate Instuctional Applications for Users with Intellectual Disability

Marian McDonnell* and Irene Connolly

Department of Technology and Psychology, Institute of Art, Design and Technology, Kill Avenue, Dun Laoghaire, Co. Dublin, Ireland

Background: Despite the abundance of literature in the area of human-computer interaction (HCI), there is limited HCI research about users with intellectual disability (ID).This research project evaluates Let's Stop Bullying, an eLearning application in development at 
the Institute of Art, Design and Technology, Dublin, Ireland. This application aims to educate young adults with mild to moderate intellectual disability about bullying and cyberbullying. Cyberbullying is bullying, when perpetrated through technology.Understanding our participants' needs and observing their interaction, within a user-centred approach, this evaluation of a web-based instructional application informed the iterative process of development of an appropriate instructional resource intervention to educate users with ID. The aim was to design an accessible and userfriendly instructional resource to empower and educate about bullying and cyberbullying. Using audio, picture-based interventions, game-based learning and scripts, this study explores the level of user engagement with the application and the preferred method of learning for participants with ID.

Method: Purposive sampling was used to recruit participants of unspecified levels of ID. Recruitment was obtained in accordance with ethical standards of the Psychological Society of Ireland (PSI). Inclusion criteria specified an ability to communicate verbally and being over 18 years of age. These criteria were established due to lack of additional support from a speech and language therapist. Between five to eight users with ID have been suggested to identify up to $80 \%$ of interface problems. However, twenty-two individuals with mild to moderate intellectual disability took part in our focus groups and laboratory sessions over a three week period. Seven tasks were created to explore the application, with progressing difficulty to accommodate levels of ability through four modes of learning (game, video, audio, reading). The evaluation investigated preferred learning modes and engagement for this user group. The study employed a mixed-methods design including observational and inquiry methods of usability evaluation.

Key results: The 22 participants $(9=$ male, $13=$ female), who took part in the study, were ranging in age from 19-34 years $(\mathrm{M}=22, \mathrm{SD}=3.72)$. Their use of computers included smartphone, iPad, laptop and PC. Students indicated using the computer everyday ( $n=$ $14)$, once a week $(n=7)$, or once a month $(n=$ 1). All participants indicated using a computer $(n=$ 22) with varying frequency summarised below. Participants reported using the applications Snapchat, Facebook, WhatsApp, Skype, and Facetime. Of those that use Facebook $(n=12), 75 \%$ found it easy to remember their password $(n=9)$. Adapted System Usability Scale(SUS) self- report measures indicated that participants liked the Let's Stop Bullying application. The listening activity was the preferred learning modality, followed closely by the game activity. Indications are that participants would like to see more games and videos with people in the application. Observational analysis identified occurrences of positive and negative emotional engagement. Results revealed more positive emotional responses than negative emotional responses.

Conclusion: There is a lack of suitable instructional applications for users with ID. Understanding users' identified preferences informs the process of development to bridge this ditgital divide. This helps to create appropriate instructional interventions with value for users with ID, engaging them with educational technology.

Keywords: Intellectual Disability, Instructional Technology, Cyberbullying, Usability, User Engagement.

*Corresponding author. E-mail: marian.mcdonnell@ iadt.ie

\section{The Contribution of iMovie Editing to Improve Storytelling Skills of a Student with Deafness and ADHD}

Avigail Provisor ${ }^{\mathrm{a}, *}$ and Betty Shrieber ${ }^{\mathrm{b}}$

${ }^{a}$ M.Ed program in Educational Technology, Kibbutzim College, Tel-Aviv, Israel

${ }^{\mathrm{b}}$ Program Director M.Ed program in Educational Technology, Kibbutzim College, Tel-Aviv, Israel

Background: This case study examined the contribution of video modeling and processing it, via iMovie editing, to promote planning and organizational skills in storytelling, of a student with deafness with Attention Deficit Hyperactive Disorder (ADHD), while describing his own personal experience in Sign Language. Deaf and Hard of Hearing (DHH) students with ADHD show lower test scores in assignments that require maximum utilization of executive skills, for example telling a personal story (Cannizzaro \& Coelho, 2013).

In the past few years, researchers have been increasingly interested in the ability of DHH students to express their narrative. It has been found that students with deafness show lesser ability than their hearing peers in the structural aspect of the narrative discourse. Sometimes the difficulty stems from the differences between sign language and written language (Rathmann, Mann \& Morgan, 2007).

Method: Manny is a deaf student with attention deficit disorder who studies in a special needs school. Dur- 
ing a composition lesson, Manny was required to share his personal experiences with his classmates. This task was very difficult for him and his stories lacked in sequence. The rest of the students found it difficult to understand him and as a result their attention level decreased. When Manny noticed this, he would give up and return to his place. This affected his sense of competence and the level of participation in the lesson. A qualitative approach to a case study was conducted. An intervention program was developed that integrates video modeling and video processing using the iMovie application. According to this method, Manny watched his video telling an experience story, then edited the video and finally watched it again so that he can learn what a correct narrative scheme is.

Key results: The findings show that the video Modeling served as a mirror for the student and reflected on his conduct during storytelling of his personal experience, to his classmates. In addition, as a result of the editing process, the student internalized the narrative scheme while maintains a temporal sequence and using connecters in a correct order. The processes indicates impressive progress of the student's of executive control of information and to his ability to initiate the editing process without the teacher mediation. In addition iMovie was found as an intuitive app for the student. It enabled him to implement the editing technique easily, thus facilitating the learning process of the narrative schema.The findings did not show the contribution to the student's planning and flexibility skills, nor to the extension of his attention range.

Conclusion: Children with deafness usually rely on their sight in order to absorb various experiences and information. Thus, watching his video before and after the editing process helped Manny to see easily where his difficulties lay and facilitated the learning process. Moreover, it helped him correct easily his errors, improving his control skill while internalizing the narrative schema. There is still a need for continuing practice in order to enhance the level of the story that is being told.

Keywords: Executive functions, Video modeling, Imovie, Storytelling, Deaf and Hard-of-Hearing people (DHH).

*Corresponding author. E-mail: avigailprovisor@ gmail.com; betty.shrieber@smkb.ac.il

Observation of HandiMathKey Appropriation Phase by Disabled Students in a Middle School Frédéric Vella $^{\mathrm{a}, *}$, Nathalie Dubus ${ }^{\mathrm{b}}$, Christine Gallard ${ }^{\mathrm{b}}$,
Cécile Malet ${ }^{\mathrm{b}}$, Véronique Ades $^{\mathrm{b}}$ and Nadine Vigouroux $^{\mathrm{a}}$

a IRIT, UMR CNRS 5505, Paul Sabatier University, 118 Route de Narbonne, 31062 Toulouse Cedex 9, France ${ }^{\mathrm{b}}$ ASEI, Jean Lagarde center, 1 Avenue Tolosane, 31520 Ramonville-Saint-Agne, France

Background: The mathematical input in text editors by disabled students is demanding both at the functional level (motor disorder) and at the cognitive level (attention, visual-spatial, memory) and generates fatigability little productive and effective gain. To reduce these demands, HandiMathKey, a mathematical keyboard software was designed by applying a usercentered method. The aim of paper is to report how different disabled students have appropriated HandiMathKey in a middle school by an observation carried out by a multidisciplinary team. The hypothesis is that HandiMathKey can be learned and used by all students with disabilities.

Method: A team (one mathematics teacher, one occupational therapy and one specialized education assistant) leads an interdisciplinary workshop to observe how the students accept and use HandiMathKey. This field study has started since September 2018 at the Jean Lagarde rehabilitation and education Center.The HandiMathKey was proposed to 23 students (19 with hand motor impairment, 3 with visual impairment and 1 other with dyspraxic and dysgraphic disorders) in three classrooms of middle school. Two classrooms use the HandiMathKey with the Microsoft Office editor, the third with the one Libre Office. Every three weeks for each class, a workshop takes place during the class time: the teacher introduces the HandiMathKey features corresponding to the mathematical concepts being taught; then the students are invited to do mathematical exercises and the occupational therapist may assist them in getting to grips with the HandiMathKey. During the workshop two types of data are recorded: 1) notes of teacher and occupational therapist within reflection diary; 2) activity log of the use of HandiMathKey. After five workshop sessions the students are invited to reply to answer a SUS (System Usability Scale questionnaire).

Key results: This study confirms the interest of having a multidisciplinary team for observation and assistance in the appropriation phase of HandiMatHKey. We report the five observations of 9 students with motor impairment. The students were all volunteers but during these workshops they did not seen the immediate interest of HandiMathKey. The students appro- 
priate HandiMathKey due to the interface affordance. However, there is a need to learn how to use the LibreOffice text editor. The typing with HandiMathKey and LibreOffice is similar to reading the mathematical formula, which makes it more affordable for students with planning and visual-spatial difficulties. As the sessions progressed there is a greater participation of students.

Conclusion: This field study shows that the appropriation phase of HandiMathey is necessary for students to agree to use it as an assistive technology. Another perspective is to analyze the typing speed and the error rate.

Keywords: Mathematical Virtual Keyboard, Acceptability, disabled students, observation, education.

${ }^{*}$ Corresponding author. E-mail: Frederic.Vella@irit.fr

\section{Digital Assistive Technology Education and Train- ing \\ Annalu Waller* \\ Computing, School of Science \& Engineering, Univer- sity of Dundee, Perth Road, Dundee DDI 4HN, Scot- land, $U K$}

Background: Assistive Technology (AT) is, by its nature, interdisciplinary. However, education and training in AT is usually delivered within specialist programmes, e.g., in occupational therapy where AT is intrinsic to the delivery of services which aim to support independence and inclusion of persons with disabilities. With the increase in digital AT, there is a need to equip a wider range of professionals with knowledge and skills to ensure effective use of such technology. Training in digital AT and accessibility has been embedded into both mainstream undergraduate and taught postgraduate degree programmes in Computer Science at Dundee University. This study was conducted to identify the range and focus of AT training as reported in the literature, compared to that being offered in Dundee.

Method: A high sensitivity literature review was conducted to identify the nature and delivery of AT training. The Web of Science bibliographic database was used to identify published outputs using the terms: (assistive technology) AND (teaching OR learning OR education OR training OR instruction OR "professional development"). A total of 1,504 papers were returned. Examination of the paper titles revealed a focus on the teaching or instruction of users instead of professional education and training. The search was thus refined by using the following terms: (assistive tech- nology) AND (education OR "professional development"). On inspection, 32 papers describing a range of AT education and training.

Key results: The papers described a range of education and training of AT. Some programmes were specifically designed to provide training to a multidisciplinary cohort of students at post-graduate level. Such programmes tended to be Biomedical with AT as a component, while some have focused specifically on AT, equipping students from both a technical and a clinical background to develop skills in the assessment and provision of AT. Training and awareness of AT was also provided within specialist professional training, e.g., teacher training and occupational therapy, although there is evidence that special educational teachers and therapists lack sufficient exposure to AT.

Conclusion: Most AT training and accreditation have a medical and healthcare focus. This may be appropriate for traditional AT which is dependent on biomechanical and electronic engineers to provide access to mobility (wheelchairs, prostheses, etc.) and aids for daily living (hoists, kitchen appliances, etc.). However, computer science and software engineering are now at the forefront of developing inclusive and accessible technology. Accessible mainstream computing and digital AT have the potential to enable people with complex physical and/or intellectual disabilities to access a wide range of activities including communication, education, employment and recreation. In order to ensure that the "digital economy" does not result in an ever increasing "digital divide", digital AT and accessibility must be embedded into mainstream software engineering curricula. It can be argued that instead of viewing accessibility as an 'optional extra', this area of technological design and application should be embedded into mainstream degree programmes, thus equipping software engineers to meet the needs of any individual, recognizing the natural diversity in all societies.

Keywords: Accessibility, Assistive Technology, Augmentative and Alternative Communication, Digital Assistive Technology, Higher Education, Training. *Corresponding author. E-mail: a.waller@dundee.ac. uk

\author{
A special Needs Course in Undergraduate Health \\ Professions: An Evaluation Using Mixed Methods \\ Joaquim Alvarelhão ${ }^{\mathrm{a}}$, Alexandra Queirós ${ }^{\mathrm{a}}$, Margarida \\ Cerqueira $^{\mathrm{b}}$, Anabela G. Silva ${ }^{\mathrm{b}}$ and Nelson P. Rocha ${ }^{\mathrm{c}, *}$

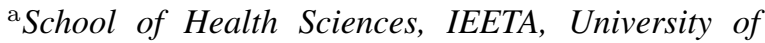


Aveiro, Campo Universitário de Santiago, 3810-193 Aveiro, Portugal

${ }^{\mathrm{b}}$ School of Health Sciences, CINTESIS, University of Aveiro, Campo Universitário de Santiago, 3810-193 Aveiro, Portugal

${ }^{\mathrm{c}}$ Department of Medical Sciences, IEETA, University of Aveiro, Campo Universitário de Santiago, 3810-193 Aveiro, Portugal

Background: Assistive technology as a strategy to improve functioning of persons with disability is commonly accepted as of high value. It is an area where different professional specialties converge (e.g. engineering and health), but the theme has a narrow presence in academic syllabus among graduation programs. Despite the impact of this strategy on the level of participation for people with disabilities, the fact is that health professionals still demonstrate a lack of positive attitude towards assistive technology and a lack of training for its counseling or prescription. The aim of this study was to evaluate the impact of the Special Needs Course (driven for assistive technology) in undergraduate students from the School of Health Sciences - University of Aveiro, Portugal. This course if framed by the International Classification of Functioning, Disability and Health (ICF) conceptual model.

Method: Sequential mixed methods were used based on the first three levels of Kirkpatrick Model of Training Evaluation. From the nursing graduation program, 32 students (26 female) and aged between 18 and 24 years participated in the first two levels of the study. For the third level four health professionals were recruited (three female), aged between 28 and 32 years and with more than five years of experience, who had attended the course during graduation. Concerning the first level of the Kirkpatrick Model ('Reaction'), the results from the questionnaire (comprising eight items; 1-9 likert scale, $9=$ higher quality) used in Quality Management System of University of Aveiro were analyzed. The second level ('Learning') achieved by students was assessed using the pre- (day 1) and posttraining (last session) test, containing five questions on the content addressed and five questions on contents not addressed but related to the theme. Finally, the third level ('Behavior') was assessed through a focus group. Data analysis included descriptive and inference statistics for the first two levels and content analysis for the third level.

Key results: The global median for 'Reaction' was Med = 7 (IQ1-IQ3 = [6.5-7.5]), with the highest values found in question about 'availability of study mate- rials' and lower values found in 'articulation between the activities developed and the previous competences. The repeated Anova measures show a significant effect for the difference between pre- and post-training in addressed questions when compared with the difference for not addressed questions $(\mathrm{F}(1,31)=6.4$, $p<0.05$ ). Focus group revealed: (i) the content of the course is considered important for those that work with persons with disabilities or elderly persons in community but less for those who work on hospital settings; (ii) ICF approach improves clinical reasoning for 'enabling' but professional contexts still show reluctance in adopting it as a framework for practice; (iii) a multidisciplinary approach should be implemented during practical classes.

Conclusion: The Special Needs Course is an applied model for integrating assistive technology in health professionals' education. Although the students reveal a lack of previous experiences in approaching these themes, the results suggest a good adherence and learning of the content addressed. The real impact of this course on professional competencies and consequently on health interventions should continue to be deepened.

Keywords: Special needs course, Graduation program, Assistive technology, ICF.

${ }^{*}$ Corresponding author. E-mail: npr@ua.pt

The Impact of Using Learning Apps on Executive Functions: Task Initiation and Persistence of Students with Attention and Learning Disorder

Betty Shrieber

Program Director of M.Ed program in Educational Technology at Kibbutzim College of Education, Technology and the Arts, Tel-Aviv, Israel

Background: The presentation will describe seven studies that have been examining the impact of using learning apps: iPad apps and learning software, to enhance executive functions: task initiation and persistence among students with ADHD (Attention Deficit Hyperactive Disorder), SLD (Specific Learning Disorders), and challenging behavior students, characterized with difficulties in emotional regulation.

Initiation and completion of academic tasks pose the most common difficulties for pupils with learning difficulties. Task initiation is defined as the ability to independently begin a task when someone wants or is instructed to do so (Anderson, 2002; Gioia, Isquith, $\&$ Guy, 2015). Task persistence or sustained attention 
refers to continuous performance of a task without distraction in order to complete the task (Barkely, 1997). Difficulties in sustaining working memory have a negative impact on one's ability to remain attentive and focused for appropriate lengths of time (Isquith, Gioia, Guy, Kenworthy \& Staff, 2008).

Method: Seven single-subject research design were used for this study. An ABAB design, with a withdrawal between Phase B1 and Phase A2, was implemented (Cooper, Heron, \& Hewar, 2007; Kennedy, 2005).

Subjects span a range of backgrounds, including age, school, and different learning disorders: ADHD, challenging behavior students and students with SLD. Each individual is separately exposed to a series of lessons under controlled conditions (baseline) and a series of lessons under experimental conditions (intervention). The data provide a framework for describing the changes in the use of single-subject designs for each student.

Phase A was used to establish a baseline and to monitor the use of traditional materials, while during the intervention phase (B), teachers used a portable computer or iPad with learning apps, including camera and dynamic interactive games.

Key results: The graphed data indicated a clear difference between Phases A and B across all participants and across all variables. The means showed similarities within phases and a clear difference between Phases A and $\mathrm{B}$.

The visual examination results suggest a decrease average of time response to the assignments (Task initiation) along with reducing the average number of breaks during the intervention phase (task persistence), when use a laptop or iPad with learning apps. Also, the changes in levels for all studies occurred immediately after the change in phases. Phase B2 level show stability in the graph variation and/or data reduce, for all participants.

Conclusion: Children with ADHD, SLD, and challenging behavior, have hard time to initiate tasks and keep attention persistence during learning assignments. The study indicates that using learning apps allows these students to engage their assignments and extends the duration of attention throughout the task.

Using learning apps along with assistive technology, helps raise children interest in learning, provides instant feedback, and increases the students' accessibility (as with reading software) to challenging material. When students succeed to accomplish tasks as a result of their hard work, they feel empowered as they rec- ognize that their persistence will lead to improved academic performance (Meltzer, Dunstan-Brewer \& Krishnan, 2018).

Keywords: Specific Learning Disorders, Executive functions, (Learning apps), (Task initiation).

*Corresponding author. E-mail: betty.shrieber@smkb. ac.il

\section{“ADAPEI transport": A Learning and Navigation App for Young Adults with Intellectual Disabilities to Improve their Autonomy to Take Public Trans- port}

Jesus Zegarra Flores ${ }^{\mathrm{a}, *}$, Gaëlle Malnati ${ }^{\mathrm{b}}$, Jean-Jacques Stevens ${ }^{\mathrm{b}}$, Nadia Laayssel ${ }^{\mathrm{b}}$, Gilbert Geneviève ${ }^{\mathrm{b}}$, Jean-Baptiste de Vaucresson ${ }^{\mathrm{b}}$, Remi Coutant ${ }^{\mathrm{b}}$ and Jean Pierre Radoux ${ }^{\mathrm{a}}$

a'Medic@,Département de recherche d'Altran, Parc d'Innovation, Boulevard Sébastien Brandt, Bât Gauss, CS 20143, 67404 Illkirch, France

${ }^{\mathrm{b}}$ ADAPEI du Territoire de Belfort, 11 route de Phaffans, Roppe 90380, France

Background: This project is done in collaboration between Altran and ADAPEI from Belfort (association for helping young adults with intellectual disabilities). Problems can happen to disabled young adults when they want to go from a departure point to the final point taking the public transport because of the complexity of the transport network. The aim of our work is to develop a learning and navigation app to improve their autonomy for taking public transport with a menu to save paths and another to navigate. In the state of the art, an adapted navigation app called Way2B has been found.

Method: Our app has been developed in an Android system. To create a path, all the peripheries of the Phone have been used: for example, the camera to take a photo from every landmark, the microphone to record the actions to do, the keyboard for typing the actions to do. Pictograms associated to the actions, the line, name and time schedules of the bus can also be added. GPS coordinates are also saved in every landmark. While saving the path, GPS coordinates are also recorded every second. This information is used to visualize sequentially, in a map the path done with the waypoints. Three main functions based on data fusion have been programmed to help the children:

- To prevent the child to press on the stop button in the bus 
- To indicate the direction to take when they get off from the bus.

- To anticipate the change of directions in pedestrian navigation.

Tests were done with specialists who used the app for the first time to record 2 adapted paths. Additionally, 2 children with medium level of disability and medium experience taking public transport (always accompanied by a specialist) tested the functions of the app in 2 hybrid paths (bus and pedestrian mode) accompanied by a researcher and a specialist.

Key results: After using the app for recording a path with 4 steps and another with 8 steps, specialists think that the app is intuitive and easy to use and they can create adapted paths in a fast way.

The alarms for preventing to press the stop in the bus were understood by the children and automatically they pressed on the button. The information on the map like the current position and the arrows for turning right or left seem to be understood by the children after explaining them the meaning. While they were doing the pedestrian path we have asked them what actions to do when arrows appear and they answered correctly. Conclusion: We have shown the efficiency of ADAPEI transport having encouraging results. It has been tested the efficiency in the creation of the path having good results and acceptance with new end users. Children have understood different information like alarms and images that appears on the screen during navigation confirming their accuracies. Future work will include adding information from bus time schedules in real time and more tests with children to validate the functions.

Keywords: GPS, bus, transport, navigation, intellectual disability.

*Corresponding author. E-mail: jesus.zegarraflores@ altran.com

\section{Assessment of SlideWiki OpenCourseWare Plat- form by Individuals with Mild or Moderate Intel- lectual Disability}

Sophia Karagouni ${ }^{\mathrm{a}}$, Elli Kafritsa ${ }^{\mathrm{b}, *}$, Maria Mouka ${ }^{\mathrm{c}}$ and Dimitris Tourlidas ${ }^{\mathrm{d}}$

a,b,c,d Research \& Development Department, Margarita Vocational Training Center, Mesologiou 4-6 \& Redestou 7-9, Nea Penteli, 152 36, Athens, Greece

Background: The introduction of new software does not often take into account the opinion of people with intellectual disability (ID) and issues, such as acces- sibility, usability and inclusive design are often neglected or left out. The purpose of this research was to train people with ID in order to use the SlideWiki OpenCourseWare (OCW) platform and examine the extent to which it is accessible to this population. Recent research indicates that SlideWiki, which among others allows the creation of highly-structured remixable and easily shared OCW, is a promising addition in the field of web-based learning. However, specific adaptations are needed, such as easy-to-read description of the platform functions so as to be fully accessible to learners with ID.

Method: The sample of this study $(n=10)$ consisted of 4 women and 6 men with mild and moderate ID, aged from 20 to 50 years old, attending a vocational education and training center in Greece. The training and assessment period lasted from 6 to 8 weeks depending on the group of beneficiaries. The hourly training sessions took place from once to twice a week and occurred almost concurrently with the platform assessment. The training tools used were presentations uploaded in the platform in an easy-to-read format and an easy-to-read manual in printed form. Presentations created by the participants in topics that interested them were also used. The participants assessed the accessibility of the initial page and the easiness of the search function, the profile creation and signing in. They also evaluated their understanding of the text in the screen, the platform settings, the use of colors, text functions, pointer and other functional characteristics and they were able to manage their presentations. The trainers observed some initial difficulties in the handling of the platform by the participants, although eventually they became accustomed with some features of the new edition. In order to develop a more comprehensive understanding of the data, triangulation was used. The participants completed 3 forms at the end of the training period in order to record their exact opinions and facilitate the data quantification.

Key results: The different forms provided divergent results, regarding the accessibility and easiness of the platform. Moreover, the opinion of the trainers on how the beneficiaries perceived the use of the software was not always identical with the opinions recorded in the forms. The most important finding though, was that the beneficiaries prided themselves on working as a team and having their opinion taken into consideration.

Conclusion: The suggestions that were put forward by the beneficiaries are worth mentioning, as these could make the platform more usable and convenient to the people with ID. Some key suggestions include the 
translation of the platform, the change in the appearance of certain functions (e.g. arrows, pointer) and the use of speech-to-text option which might be more convenient than text-to-speech specifically for this population.

Keywords: SlideWiki, Intellectual Disability, OpenCourseWare.

*Corresponding author. E-mail: inclusion@eeamargar ita.gr

\section{Can Accessible Technology Help Person Centered Planning? Exploring the Role of an ICT Solution to Evidence Value in Service Delivery for People with Intellectual Disabilities (ID)}

Sarah Gavra Boland ${ }^{\mathrm{a}, *}$, John Owuor ${ }^{\mathrm{b}}$, Alan Byrne ${ }^{\mathrm{c}}$ and Peter Gallagher ${ }^{\mathrm{d}}$

${ }^{a}$ Liffey Services, Saint John of God Community Services clg, Cookstown Way, Tallaght, Dublin 24, Ireland ${ }^{\mathrm{d}}$ Saint John of God Hospitaller Services, Stillorgan, Co. Dublin, Ireland

Background: Under 'New Direction Guidelines' from the Health Services Executive (HSE) Ireland, there are pointers to how the implementation of a digital environment can support and ensure a more efficient and effective service. However, health and social service providers face persistent challenges such as budget limitations and inadequate staffing levels. Other challenges include compliance to national, and international regulations such as HIQA (Ireland) UNCRPD, Accessibility ACT and GDPR. All these affect the ability of services to invest in and manage digital environments required to support efficient and effective services. Saint John of God, Liffey Services have engaged in a pilot project with Asperico the developers of iplanit, a cloud based person-centered client management system. It supports person centered provision by keeping track of client's goals, focusing on outcomes and giving the user easy access to their information. The project builds on a previous one which explored how Assistive Technology (AT) can be used to support independent living and the adoption and development of accessible digital personal plans.

Method: The pilot project is complemented by a Strauss and Corbin's grounded theory study that uses an interpretive approach, underpinned by symbolic interactionism as the theoretical framework, to gain insights into the participants' narratives on how iplanit mediates person-centered planning. This qualitative study is split into three phases; i) desktop study phase with literature review, ii) pre-pilot interviews and, iii) a post-pilot focus group discussions. The pre-pilot interviews were carried out between May 2018 to April 2019. A total of 34 participants were interviewed for phase two including 12 persons with ID, 6 family members, 10 support staff, 6 management and community members.

Key results: Data analysis found that accessible digital personal plans facilitated plan owners to feel empowered, and a sense of ownership over their information and plans. iplanit has provided better accessibility to relevant information for all stakeholders. It gives the plan owner autonomy over the development of their plans, a direct say in what they would like to achieve evidenced through the use of accessible multimedia. For service providers, the system provides an accessible platform to evidence personal plans, related activities and outcomes. Findings also indicate reduced need for administrative resources compared to paper-based planning. It supports the move to community service provision. There was also evidence of improved information sharing and access between relevant stakeholders. Used effectively, iplanit can improve accountability for staff and management and provide value for money evidence in investing in digital solutions.

Conclusion: Accessible digital solutions can support person-centered planning and improve service efficiency and effectiveness if the solutions are codesigned with relevant stakeholders. However, codesigning digital solutions can be challenging. Technology developers can be reluctant to provide the necessary built-in assistive technology needed to ensure that all stakeholders can engage in the solution. Issues of copyright and public good versus profitability can also be difficult to manage in co-design processes. Further research is required to explore how best to involve people with ID and different interest stakeholders in co-design of digital solutions.

Keywords: Person Centered Plans, Accessible Technology.

*Corresponding author. E-mail: sarah.boland@sjog.ie

Evaluating a Social Inclusion Intervention with Support of a Mobile Application among Young Adults with Intellectual Disabilities

J.S. Louw* and G. Leader ${ }^{\text {b }}$

${ }^{\mathrm{a}}$ Trinity College Dublin (TCD), School of Nursing and Midwifery, Dublin, D02 T283, Republic of Ireland

${ }^{\mathrm{b}}$ National University of Ireland, Galway (NUIG), School of Psychology, H91 TK33 Galway, Republic of Ireland 
Background: Social inclusion has been regarded as a key outcome in the general health of all individuals and it has been reported to have a number of benefits, particularly for young people with intellectual disabilities (ID). Research on social inclusion and the use of digital technology among young adults with ID are reported to play a vital part in how they can connect socially. Too often, young people with ID are excluded from taking part in social activities because they struggle to understand social cues and respond appropriately. Therefore, this pilot study investigated a social inclusion intervention with the support of a mobile application to improve social interaction skills for young people with ID.

Method: A pre-post study design was adopted to evaluate the implementation of a social inclusion intervention with the support of a mobile application. In addition, three social validity questions were included at the end of each social activity that was uploaded on the mobile app. Ten participants with ID, 18 years and older, participated in a weekly intervention session using the mobile app over a period of 12 weeks. Participants were recruited from four institutions that provide day services to people with ID. Seven staff members participated with the service users to implement the intervention. The staff completed six intensive training sessions on how to use the mobile app. Parents, the ten young people with ID and staff provided input on the prototype of the mobile app prior to the intervention being implemented.

Key results: Altogether $95 \%$ of participants were diagnosed with a moderate intellectual disability and only $50 \%$ of participants managed to complete all the 6 social inclusion activities selected prior to the intervention being implemented. Examples of social inclusion activities included listening to music, swimming, shopping and taking a walk. The social interaction skills aligned with the selected social activity included a focus on listening skills, eye contact and communication skills. Staff uploaded 3 to 6 prompts as a step-by-step guide for each activity. The majority of participants reported the prompts as helpful in completing their social activities. Personalized pictorial images were uploaded on the mobile app for each activity and this encouraged and enabled participant interest. The majority of participants $(96 \%)$ reported acceptance of the use and support of the mobile app when participating in social activities.

Conclusion: The mobile app was regarded as valued when used to help participants with improving their communication skills. More importantly, the significance of an intervention that used the support of a mobile app was seen as a unique facilitator to help communicate between staff and parents on how best to support young adults with ID at home and similarly at the day service. The findings of this pilot indicate that increased opportunities where participants can practice and participate in social interaction skills with the use of assistive technology are most needed. This will result in improving self-confidence and a sense of independence for young adults with ID when they engage in real-world social environments.

Keywords: Social Inclusion, Youth, Technology, Communication.

*Corresponding author. E-mail: louwj@tcd.ie

\section{Using High Fidelity Work Simulator for Vocational Orientation, Skill Assessment and Development of Young Adults with Disabilities \\ Márk Ágoston Pulay ${ }^{\mathrm{a}, *}$ and Erika Jókai ${ }^{\mathrm{b}}$ \\ ${ }^{a}$ Budapest University of Technology and Economics, Department of Ergonomics and Psychology, Budapest, Hungary \\ ${ }^{\mathrm{b}}$ Óbuda University, Budapest, Hungary}

Background: Work is crucially important for people with disabilities as it fosters their quality of life. Many studies focus on the assessment of vocational aptitude of people with different kinds of disabilities but most of them are qualitative research; paper based tests and simple observations. However, beside those technics our main focus is already on the accurately measurable functions that remained intact and therefore can be the basis of appropriate work skills. Hence, we used a sophisticated and complex high fidelity work simulator (WS) that has the necessary broad spectrum of evaluation test batteries. This research is a part of a complex vocational orientation program in Hungary for teenagers and young adults with disabilities. The main objective of our research was to create a methodology using a reference database to support aptitude assessment and work diagnostics of teenagers and young adults with disabilities to helping vocational school and career choices, labor market orientation or the assessment of specific job skills.

Referring to the well-known Human Activity Assistive Technology (HAAT) Modell - of which the occupational performance is an important element - it describes how a person performs an occupation. Assistive technology (AT) in a broader sense - includes any 
assistive, adaptive, and rehabilitative devices for people with disabilities in order to promote greater independence by enabling them to perform tasks.

Method: The WS consists three measuring panels, as independent workstations. P1. for measuring static and dynamic forces in standing position, P2 for measuring holding/grasping forces, touching/tactile functions, and fine motor abilities in sitting position, P3. for measuring work endurance/loadability, monotony susceptibility in complex workflows. This full scale examination consists 36 different task situations each with 219 corresponding performance parameters, altogether totalling up to 203 .

We compared the values of all these 203 measured parameters between the group of 179 "healthy" persons and the sample of the 100 "disabled" young (13-30 years) persons to determine which parameters are significantly different between these categories. We used mostly the One-Sample T-Test to compare each "disabled" sample mean with the corresponding "healthy" sample means as fixed numbers. A rather strict significance level of 0.01 was set instead of the usual 0.05 .

Key results: We have found many statistically significant differences between groups. There are many remarkable overlaps of the "healthy" and "disabled" distributions: there were many "disabled" persons who performed better in certain parameters than the average of the "healthy" persons. Also there were many "healthy" persons whose performance was worse in certain parameters than the average of the "disabled" persons.

Based on the results of a work diagnostic measurements, we can give a feedback to students, career advisors or HR managers about the prospective employee's functional workability: which skills are outstanding, average, or need to be improved for the desired profession, career or job.

Conclusion: This broad variety of task situations and the vast repertory of corresponding measurable performance parameters of the WS makes it especially appropriate for skill assessment, skill development and also for vocational aptitude tests of different kinds of disabled persons.

Keywords: Work Simulator, Vocational Orientation, Skill Assessment,

*Corresponding author. E-mail: pulaymark@erg.bme. hu 